\title{
Group Multi-Attribute Decision Making Based on Interval Neutrosophic Sets
}

\author{
Amir Hossein NAFEI ${ }^{1}$, Wenjun YUAN ${ }^{1 *}$, Hadi NASSERI ${ }^{2}$ \\ ${ }^{1}$ Department of Mathematics and Information Science, Guangzhou University, Guangzhou,510006, China \\ Amir.nafei@e.gzhu.edu.cn,wjyuan1957@126.com (*Corresponding author) \\ ${ }^{2}$ Department of Mathematics and Big Data, Foshan University, Foshan, 528000, China \\ nhadi57@gmail.com
}

\begin{abstract}
This paper presents a new method for group multi-attribute decision-making (GMADM) based on interval neutrosophic sets, where decision makers determine the weights and the evaluating values of the attributes with respect to the available alternatives by using interval neutrosophic values. In comparison with other existing methods involving group multi-attribute decision making, that only consider crisp or incomplete information, the proposed method, based on interval neutrosophic sets, can handle not only incomplete information but also indeterminate and inconsistent information which is common in real-world situations. Therefore, the method presented in this paper can be more effective and efficient than other decision-making methods.
\end{abstract}

Keywords: GMADM, Decision-making, Interval neutrosophic sets, Interval neutrosophic values, Neutrosophic sets, MAGDM.

\section{Introduction}

Decision-making with an emphasis on multiattribute decision making is a significant and fundamental part of our everyday life. If the number of decision-makers and their objectives is increased while their priority becomes higher, the decision-making process is not always easy.

Because of the ambiguity of people's thinking and the complexity of objectives, it's not always possible to use crisp data. Determining the amount of uncertainty is always a major challenge in decision making. The fuzzy sets theory which is proposed by (Zadeh, 1965) is a practical approach to overcoming uncertainty, as such, it assigns membership function to any non-deterministic event. Sometimes because of uncertainty determining the degree of membership isn't possible. For this reason, (Zadeh, 1975) by considering the degree of membership as an interval value proposed interval-valued fuzzy sets (IFSs) to express the uncertainty in the membership function. (Attanasov, 1986) by adding the degree of non-membership introduced another extension of fuzzy sets namely intuitionistic fuzzy sets. The degree of elements belonging to an intuitionistic fuzzy set is represented by the membership and the non-membership degrees in $[0,1]$, respectively.

In this respect, (Atanasov \& Gragov, 1989) proposed a generalization of the intuitionistic fuzzy sets called Interval-Valued Intuitionistic Fuzzy (IVIF) set, where the membership value and the non-membership value of any of its elements are represented by an interval value of $[0,1]$.
(Smarandech, 2004, 2005) proposed Neutrosophic Sets (NSs) which are a generalization of the classical sets, fuzzy sets, and intuitionistic fuzzy sets, which provide a powerful tool for dealing with the indeterminacy. In Neutrosophic sets, a given set $A$ is described by truth, indeterminacy and falsity subsets.

Recently, NSs have become an important research topic and attracted much attention. (Wang et al., 2010) proposed a neutrosophic set which is single-valued from a scientific and engineering point of view, as an instance of the neutrosophic set. Also, (Wang et al., 2005) presented Interval Neutrosophic Sets(INSs) in which the truthmembership, indeterminacy-membership, and falsity-membership were extended to interval numbers and provided the set-theoretic operators and various properties of INSs.

A lot of papers about multi-attribute decision making and the methods for dealing with them have been published. In this way to assess the risk of bridge failure (Wang \& Elhag, 2007) introduced a decision-making method based on fuzzy group recommendations. this model enables decisionmakers to express the opinion individuality by using linguistic terms instead of precise numerical values. in addition, they introduced two-layer algorithms to evaluate the values of bridge risk factors. (Pérez, Cabrerizo \& Herreraviedma, 2010) presented a mobile decision support system for dealing with dynamic group decision-making problems. (Smolikova \& Wachowiak, 2002) 
compared aggregation techniques and analyzed different aggregation methods for group decisionmaking problems. (Ben-Arieh \& Chen, 2006) presented a linguistic labels aggregation operator, called the fuzzy linguistic order weighted average operator, and applied it for autocratic decision making using group recommendations. (Chen \& Tsai, 2015) present-ed an autocratic group decision-making method based on the ordered weighted average operator and the correlation coefficient between the preference order of the alternatives for all experts and the preference order of the alternatives for each expert, where linguistic preference matrices are used to express experts' opinions. (Parreiras et al., 2010) presented a flexible consensus scheme for multicriteria group decision making under linguistic assessments. (Pedrycz \& Song, 2011) pointed out that information granularity is an important and useful asset that helps to reach consensus in group decision making. (Kou et al., 2017) presented an optimization model for the obtaining group preference employed in AHP group decision making that is relative to the AIP with pre-defined fixed weights. (Rădulescu. C. \& Rădulescu. I., 2017) by varyi-ng the parameter $\rho$ in the Minkowski distance presented an extended Topsis approach for ranking cloud service providers.

This research presents an applied method for decision-making with group recommendations based on interval neutrosophic sets, where decision makers determine the weight and the evaluating value of each attribute by using interval neutrosophic values. Section 2 briefly outlines neutrosophic sets, interval neutrosophic sets, operational rules between INSs, score and accuracy functions and ranking method of INSs. In section 3 the above-mentioned method is extended to ranking the alternatives. In section 4 one uses an example for illustrating the proposed method and the conclusions are discussed in section 5 .

\section{Preliminaries}

This section presents a brief study of certain preliminaries concerning neutrosophic sets, interval neutrosophic sets and some other details about them.

Definition 1 (Smarandache, 1999): Let $X$ be a universe of objectives and $x \in X$. A Neutrosophic Set (NS) $N$ through $X$ can be characterized by a truth-membership function $T_{N}(x)$, an indeterminacy-membership function $I_{N}(x)$ and a falsity-membership function $F_{N}(x)$, all of them being standard or non-standard subsets of $] \mathrm{O}^{-}, \mathbf{1}^{+}[$, which can be represented as $\left.T_{N}: X \rightarrow\right] 0^{-}, 1^{+}[$, $\left.I_{N}: X \rightarrow\right] 0^{-}, 1^{+}\left[\right.$and $\left.F_{N}: X \rightarrow\right] 0^{-}, 1^{+}[$. $\quad$ It is necessary to mention that there is not any restriction on the sum of $T_{N}(x), I_{N}(x)$ and $F_{N}(x)$ therefor $0^{-} \leq T_{N}(x)+I_{N}(x)+F_{N}(x) \leq 3^{+}$.

Definition 2 (Smarandache, 2005): A Neutrosophic Set (NS) $N$ is contained in the other NS $M$ if and only if,

$$
\begin{aligned}
& \inf T_{N}(x) \leq \inf T_{M}(x), \\
& \operatorname{Sup} T_{N}(x) \leq \operatorname{Sup} T_{M}(x), \\
& \inf I_{N}(x) \geq \inf I_{M}(x), \\
& \operatorname{Sup} I_{N}(x) \geq \operatorname{Sup} I_{M}(x) \\
& \inf F_{N}(x) \geq \inf F_{M}(x),
\end{aligned}
$$

$\operatorname{Sup} F_{N}(x) \geq \operatorname{Sup} F_{M}(x)$ for all $x \in X$.

Definition 3 (Wang et al., 2010): A Single-Valued Neutrosophic (SVN) set $N$ through $X$ taking the form $N=\left\{x, T_{N}(x), \mathrm{I}_{N}(x), F_{N}(x) ; x \in X\right\}$, where $X$ be a universe of discourse, and $T_{N}: X \rightarrow[0,1], I_{N}: X \rightarrow[0,1] \quad$ and $F_{N}: X \rightarrow[0,1]$ with $0 \leq T_{N}(x)+F_{N}(x)+I_{N}(x) \leq 3$ for all $x \in X . \quad T_{N}(x), F_{N}(x)$ and $I_{N}(x)$, represent truth membership, falsity membership and indeterminacy membership degrees of $x$ to $N$, respectively.

Definition 4 (Wang et al., 2005): An Interval Neutrosophic Set (INS) $N$ through $X$ taking the form $N=\left\{x, T_{N}(x), I_{N}(x), F_{N}(x) ; x \in X\right\}$ where $X$ be a universe of discourse, and $T_{N}(x), F_{N}(x), I_{N}(x) \in[0,1] \quad$ such that $0 \leq \operatorname{Sup}_{N}(x)+\operatorname{SupI}_{N}(x)+\operatorname{SupF}_{N}(x) \leq 3$ for a $11 x \in X . T_{N}(x), F_{N}(x)$ a $n d I_{N}(x)$, represent truth membership, falsity membership and indeterminacy membership of $x$ to $N$, respectively. For convenience let $x=\left(\left[T^{L}, T^{U}\right],\left[I^{L}, I^{U}\right],\left[F^{L}, F^{U}\right]\right)$ represent a value of the Interval Neutrosophic Set (INS), and call it Interval Neutrosophic Value (INV). 
Definition 5 (Wang et al., 2005): Let

$x=\left(\left[T_{1}^{L}, T_{1}^{U}\right],\left[I_{1}^{L}, I_{1}^{U}\right],\left[F_{1}^{L}, F_{1}^{U}\right]\right)$

and $y=\left(\left[T_{2}^{L}, T_{2}^{U}\right],\left[I_{2}^{L}, I_{2}^{U}\right],\left[F_{2}^{L}, F_{2}^{U}\right]\right)$

be two INVs. The multiplication and addition operations between two INVs $x$ and $y$ are defined as follows:

$x \otimes y=\left(\begin{array}{l}{\left[T_{1}^{L} T_{2}^{L}, T_{1}^{U} T_{2}^{U}\right],} \\ {\left[I_{1}^{L}+I_{2}^{L}-I_{1}^{L} I_{2}^{L}, I_{1}^{U}+I_{2}^{U}-I_{1}^{U} I_{2}^{U}\right],} \\ {\left[F_{1}^{L}+F_{2}^{L}-F_{1}^{L} F_{2}^{L}, F_{1}^{U}+F_{2}^{U}-F_{1}^{U} F_{2}^{U}\right]}\end{array}\right)$,

$x \oplus y=\left(\begin{array}{l}{\left[T_{1}^{L}+T_{2}^{L}-T_{1}^{L} T_{2}^{L}, T_{1}^{U}+T_{2}^{U}-T_{1}^{U} T_{2}^{U}\right],} \\ {\left[I_{1}^{L} I_{2}^{L}, I_{1}^{U} I_{2}^{U}\right],} \\ {\left[F_{1}^{L} F_{2}^{L}, F_{1}^{U} F_{2}^{U}\right]}\end{array}\right)$,

also, the scalar multiplication is defined as:

$$
n x=\left(\begin{array}{l}
{\left[1-\left(1-T_{1}^{L}\right)^{n}, 1-\left(1-T_{1}^{U}\right)^{n}\right],} \\
{\left[\left(I_{1}^{L}\right)^{n},\left(I_{1}^{U}\right)^{n}\right],} \\
{\left[\left(F_{1}^{L}\right)^{n},\left(F_{1}^{U}\right)^{n}\right]}
\end{array}\right), n>0 .
$$

Definition 6 (Wang et al., 2005): A score function $S$ of an INV

$x=\left(\left[T^{L}, T^{U}\right],\left[I^{L}, I^{U}\right],\left[F^{L}, F^{U}\right]\right)$

is defined as:

$S(x)=\frac{2+T^{L}+T^{U}-2 I^{L}-2 I^{U}-F^{L}-F^{U}}{4}$,

where $S(x) \in[-1,1]$.

Theorem 1. Let

$$
\begin{aligned}
& x=\left(\left[T_{1}^{L}, T_{1}^{U}\right],\left[I_{1}^{L}, I_{1}^{U}\right],\left[F_{1}^{L}, F_{1}^{U}\right]\right) \text { and } \\
& y=\left(\left[T_{2}^{L}, T_{2}^{U}\right],\left[I_{2}^{L}, I_{2}^{U}\right],\left[F_{2}^{L}, F_{2}^{U}\right]\right) \text { be }
\end{aligned}
$$

two INVs, in this case, If $x \subseteq y$, then $S(x) \leq S(y)$.

Proof. According to Definition 6, we have:

$$
\begin{aligned}
& S(x)=\frac{2+T_{1}^{L}+T_{1}^{U}-2 I_{1}^{L}-2 I_{1}^{U}-F_{1}^{L}-F_{1}^{U}}{4}, \\
& S(y)=\frac{2+T_{2}^{L}+T_{2}^{U}-2 I_{2}^{L}-2 I_{2}^{U}-F_{2}^{L}-F_{2}^{U}}{4},
\end{aligned}
$$

Therefore:

$$
\begin{aligned}
& S(y)-S(x)=\frac{1}{4}\left(\left(T_{2}^{L}-T_{1}^{L}\right)+\left(T_{2}^{U}-T_{1}^{U}\right)+2\left(I_{1}^{L}-I_{2}^{L}\right)\right. \\
& \left.+2\left(I_{1}^{U}-I_{2}^{U}\right)+\left(F_{1}^{L}-F_{2}^{L}\right)+\left(F_{1}^{U}-F_{2}^{U}\right)\right),
\end{aligned}
$$

If $x \subseteq y$, then

$T_{1}^{L} \leq T_{2}^{L}, T_{1}^{U} \leq T_{2}^{U}, I_{1}^{L} \geq I_{2}^{L}, I_{1}^{U} \geq I_{2}^{U}$ and

$F_{1}^{L} \geq F_{2}^{L}, F_{1}^{U} \geq F_{2}^{U}$,

hence,

$\left(T_{2}^{L}-T_{1}^{L}\right) \geq 0,\left(T_{2}^{U}-T_{1}^{U}\right) \geq 0,\left(I_{1}^{L}-I_{2}^{L}\right) \geq 0$,

$\left(I_{1}^{U}-I_{2}^{U}\right) \geq 0,\left(F_{1}^{L}-F_{2}^{L}\right) \geq 0$

and $\left(F_{1}^{U}-F_{2}^{U}\right) \geq 0$.

Then, it follows that $S(y)-S(x) \geq 0$ and

$S(y) \geq S(x)$.

Definition 7 (Wang et al., 2005): Let

$x=\left(\left[T^{L}, T^{U}\right],\left[I^{L}, I^{U}\right],\left[F^{L}, F^{U}\right]\right)$ be an INV.

The accuracy function of $x$ is defined as follows:

$$
\begin{aligned}
& A(x)=\frac{1}{2}\left(T^{L}+T^{U}-I^{U}\left(1-T^{U}\right)-I^{L}\left(1-T^{L}\right)-F^{U}\right. \\
& \left.\left(1-I^{L}\right)-F^{L}\left(1-I^{U}\right)\right)
\end{aligned}
$$

where $A(x) \in[-1,1]$.

Lemma 1. The accuracy function $A$ has been reduced to the accuracy function proposed by (Nayagam, Muralikrishnan \& Sivaraman, 2011) if $I^{L}, I^{U}=0$ and $T^{U}+F^{U} \leq 1$.

Definition 8 (Wang et al., 2005): Consider two interval neutrosophic values such as:

$$
\begin{aligned}
& x=\left(\left[T_{1}^{L}, T_{1}^{U}\right],\left[I_{1}^{L}, I_{1}^{U}\right],\left[F_{1}^{L}, F_{1}^{U}\right]\right), \\
& y=\left(\left[T_{2}^{L}, T_{2}^{U}\right],\left[I_{2}^{L}, I_{2}^{U}\right],\left[F_{2}^{L}, F_{2}^{U}\right]\right) .
\end{aligned}
$$

The ranking method of these two INVs $x$ and $y$ will be as follows:

1. If $S(x)>S(y)$ then $x>y$.

2. If $S(x)<S(y)$ then $x<y$.

3. If $S(x)=S(y)$ then,

$$
\text { I. If } A(x)>A(y) \text { then } x>y \text {. }
$$

II. If $A(x)<A(y)$ then $x<y$. 


\section{A New GMADM Method Based on INSs}

Assume that there are $n$ alternatives $A_{1}, A_{2}, \ldots, A_{n}, m$ attributes $B_{1}, B_{2}, \ldots, B_{m}$, and $k$ decision-makers(DMs) $D_{1}, D_{2}, \ldots D_{k}$. Assume that $E_{p}=\left(e_{i g}^{p}\right)_{n \times m}$ be an evaluating matrix of the alternatives with respect to the attributes provided by DM $D_{p}$, can be shown as follows:

$$
\begin{aligned}
& \begin{array}{llll}
B_{1} & B_{2} & \cdots & B_{m}
\end{array} \\
& E_{p}=\begin{array}{c}
A_{1} \\
A_{2} \\
\vdots \\
A_{n}
\end{array}\left[\begin{array}{cccc}
e^{p}{ }_{11} & e^{p}{ }_{12} & \ldots & e_{1 m}^{p} \\
e^{p}{ }_{21} & e^{p}{ }_{22} & \ldots & e_{2 m}^{p} \\
\vdots & \vdots & \ddots & \vdots \\
e^{p}{ }_{n 1} & e^{p}{ }_{n 2} & \ldots & e_{n m}^{p}
\end{array}\right] .
\end{aligned}
$$

where the elements of the matrix $E_{p}$ are represented by INVs.

Assume that $\bar{w}_{p}=\left(\bar{w}_{i}^{p}\right)_{1 \times m}$ be a vector of weight for attributes determined by DM $D_{p}$ that $\bar{w}_{i}^{p}$ is an INV, and $W_{p}^{(r)}$ represent the weight of DM $D_{k} \quad$ at the $\mathrm{r}^{\text {th }}$ round, where $W_{p}^{(r)} \in[0,1]$ and $\sum_{p=1}^{h} W_{p}^{(r)}=1, r \geq 1$. The method proposed in this paper for solving group multiattribute decisionmaking problems based on INSs can be presented as follows:

Step (1). Suppose that $r=1$ and $W_{i}^{(1)}=\frac{1}{k}$, $(i=1, \ldots, k)$ where $W_{1}^{(1)}, W_{2}^{(1)}, \ldots, W_{k}^{(1)}$

respectively represents the weights of the decision-makers (DMs) $D_{1}, D_{2}, \ldots, D_{k}$ in the first round. According to the multiplication operator of INSs presented in eq. 1 the weighted evaluating matrix $H_{p}$ based on the DM $D_{p}$ will be calculated as follows:

$$
\begin{aligned}
& \begin{array}{llll}
B_{1} & B_{2} & \cdots & B_{m}
\end{array} \\
& H_{p}=\begin{array}{c}
A_{1} \\
A_{2} \\
\vdots \\
A_{n}
\end{array}\left[\begin{array}{ccccc}
e^{p}{ }_{11} \otimes \bar{w}_{1}^{P} & e^{p}{ }_{12} \otimes \bar{w}_{2}^{P} & \ldots & e^{p}{ }_{1 m} \otimes \bar{w}_{m}^{P} \\
e^{p} \otimes \bar{w}_{1}^{P} & e^{p}{ }_{22} \otimes \bar{w}_{2}^{P} & \ldots & e^{p}{ }_{2 m} \otimes \bar{w}_{m}^{P} \\
\vdots & \vdots & \ddots & \vdots \\
e^{p} \otimes \bar{w}_{1}^{P} & e^{p}{ }_{n 2} \otimes \bar{w}_{2}^{P} & \ldots & e^{p}{ }_{n m} \otimes \bar{w}_{m}^{P}
\end{array}\right] \\
& \begin{array}{llll}
B_{1} & B_{2} & \cdots & B_{m}
\end{array}
\end{aligned}
$$

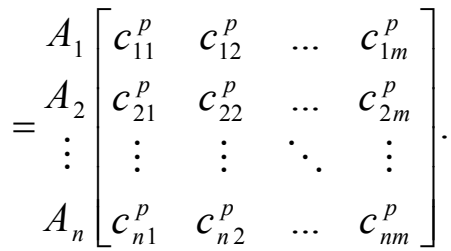

Step (2). Obtain the aggregated evaluating matrix $Z$ as follows:

$$
\begin{aligned}
& \begin{array}{llll}
D_{1} & D_{2} & \cdots & D_{k}
\end{array}
\end{aligned}
$$

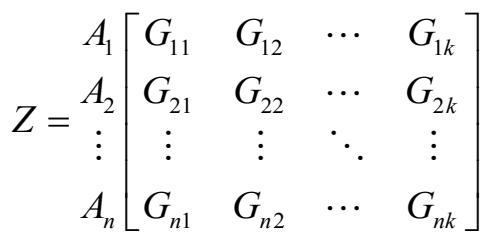

where $G_{i p}$ is an INV, representing the sum of alternatives with respect to DM $D_{p}$. Based on the addition operator $\oplus$ presented in eq. 2 the components of the matrix $Z$ can be calculated as follows:

$$
G_{i p}=c_{i 1}^{p} \oplus c_{i 2}^{p} \oplus \cdots \oplus c_{i m}^{p} .
$$

Step (3). By using the score function $S$ presented in eq. 4 and the aggregated evaluating matrix where it was obtained in the last step, the function matrix $S\left(\mathrm{G}_{i p}\right)$ can be constructed as follows:

$$
S=\frac{A_{1}}{A_{2}} \vdots_{A_{n}}\left[\begin{array}{cccc}
S\left(G_{11}\right) & S\left(G_{12}\right) & \cdots & S\left(G_{1 k}\right) \\
A_{n}\left(G_{21}\right) & S\left(G_{22}\right) & \cdots & S\left(G_{2 k}\right) \\
\vdots & \vdots & \ddots & \vdots \\
S\left(G_{n 1}\right) & S\left(G_{n 2}\right) & \cdots & S\left(G_{n k}\right)
\end{array}\right],
$$

where $S\left(G_{i p}\right) \in[-1,1], 1 \leq p \leq k$ and $1 \leq i \leq n$.

Step (4). According to the ranking method presented in Definition 8, construct the preference vector $L^{P}$ of all alternatives with respect to DM $D_{p}$ as follows:

$$
\begin{array}{rrrr}
A_{1} & A_{2} & \cdots & A_{n} \\
L^{P}=\left[\begin{array}{llll}
L_{1}^{p} & L_{2}^{p} & \cdots & L_{n}^{p}
\end{array}\right] .
\end{array}
$$

Step (5). Calculate the aggregated group evaluating value $d_{i}^{(r)}=\sum_{p=1}^{k}\left(W_{p}^{(r)} \times S\left(G_{i p}\right)\right)$ of alternative $A_{i}$ with respect to DMs $D_{1}, D_{2}, \ldots D_{k}$ where $d_{i}^{(r)} \in[-1,1]$. According to the achieved aggregated group evaluating value $d_{i}^{(r)}$, the group preference vector $Q$ of the alternatives $A_{1}, A_{2}, \ldots, A_{n}$, can be obtained as follows: 


$$
Q=\left[\begin{array}{llll}
A_{1} & A_{2} & \cdots & A_{n} \\
Q_{1} & Q_{2} & \cdots & Q_{n}
\end{array}\right],
$$

Step (6). According to the group preference vector $Q$ and the preference vector $L^{P}$, the similarity degree $S\left(Q, L^{P}\right)$ between $Q$ and $L^{P}$, can be obtained as follows:

For $p=1$ to $k$ and $i=1$ to $n$, if $Q_{i} \neq L_{i}^{p}$ then $S\left(Q, L^{P}\right)=0$

otherwise, if : $Q_{i}=L_{i}^{p}=q$

Then,

$$
S\left(Q, L^{P}\right)=S\left(Q, L^{P}\right)+[n-(q-1)]
$$

End.

And then construct the group consensus agreement degree $B^{(r)}$ of all DMs at the $\mathrm{r}^{\text {th }}$ round as follows:

$$
B^{(r)}=\sum_{p=1}^{k}\left(X_{P} \times W_{p}^{(r)}\right),
$$

where,

$$
X_{P}=\frac{S\left(Q, L^{P}\right)}{S\left(Q, L^{1}\right)+S\left(Q, L^{2}\right)+\ldots+S\left(Q, L^{k}\right)},
$$

if $B^{(r)}\langle\gamma$, where $\gamma$ is the group decisive agreement threshold value such that $\gamma \in[0,1]$ go to step 8 , otherwise the largest $d_{i}$ has the best preference order of alternative $A_{i}$.

Step (8). Let, $t_{P}^{r+1}=W_{p}^{(r)} \times\left(1+X_{P}\right)$,

and calculate the weight of DM $D_{p}$ at the $r+1^{\text {th }}$ round as follows:

$$
W_{p}^{(r+1)}=\frac{{t_{p}{ }^{r+1}}_{\sum_{p=1}^{k} t_{p}^{r+1}}}{\sum^{r}}
$$

$\begin{aligned} & \text { where } \\ & \text { step 5. }\end{aligned} \sum_{p=1}^{k} W_{p}^{(r+1)}=1$, let $(r=r+1)$ and return to The algorithm above-mentioned will be illustrated in the next section to explore the ability of the suggested method.

\section{Numerical Example}

Assume that the evaluating matrices $E_{1}, E_{2}, E_{3}$ of four manufacturing companies $C_{1}, C_{2}, C_{3}$ and $C_{4}$ as the alternatives with respect to the three

\begin{tabular}{|c|c|c|}
\hline Price Analysis & Quality Analysis & Popularity Analysis \\
\hline .950$],[0.011,0.016])$ & $[0.194,0.642],[0.091,0.517],[0.008,0.246])$ & $([0.262,0.603],[0.447,0.479],[0.181,0.308])$ \\
\hline$C_{2} \mid([0.056,0.123],[0.034,0.580],[0.287,0.329])$ & $([0.034,0.077],[0.437,0.901],[0.262,0.847])$ & $([0.500,0.570],[0.259,0.360],[0.013,0.027])$ \\
\hline$C_{3} \mid([0.406,0.718],[0.127,0.391],[0.013,0.034])$ & $([0.152,0.005],[0.193,0.576],[0.791,0.807])$ & $([0.025,0.061],[0.062,0.390],[0.130,0.300])$ \\
\hline$C_{4}([0.049,0.451],[0.314,0.672],[0.642,0.856])$ & $([0.595,0.786],[0.067,0.289],[0.411,0.498])$ & $([0.766,0.969],[0.757,0.781],[0.111,0.729])$ \\
\hline
\end{tabular}
attributes such as "Price Analysis", "Quality

\begin{tabular}{|c|c|c|}
\hline Price Analysis & Quality Analysis & Popularity Analysis \\
\hline $\mathrm{C}_{\mathrm{C}}[([0.037,0.396],[0.148,0.492],[0.148,0.258])$ & $([0.411,0.705],[0.143,0.381],[0.005,0.076])$ & $([0.294,0.650],[0.027,0.679]$ \\
\hline$C_{2} \mid([0.624,0.798],[0.035,0.711],[0.405,0.783])$ & $([0.026,0.250],[0.155,0.481],[0.834,0.881])$ & $([0.052,0.195],[0.734,0.830],[0.290,0.338])$ \\
\hline${ }^{E_{3}}={ } C_{3} \mid([0.085,0.377],[0.108,0.114],[0.900,0.965]$ & $([0.248,0.550],[0.355,0.427],[0.127,0.152])$ & $([0.427,0.496],[0.033,0.310],[0.358,0.579])$ \\
\hline$C_{4}[([0.092,0.260],[0.097,0.787],[0.011,0.512])$ & $([0.278,0.524],[0.427,0.650],[0.270,0.385])$ & $([0.090,0.401],[0.033,0.554],[0.430,0.444])$ \\
\hline
\end{tabular}
analysis" and "Popularity Analysis" given by the three decision-makers (DMs) $D_{1}, D_{2}$ and $D_{3}$ are represented as follows:

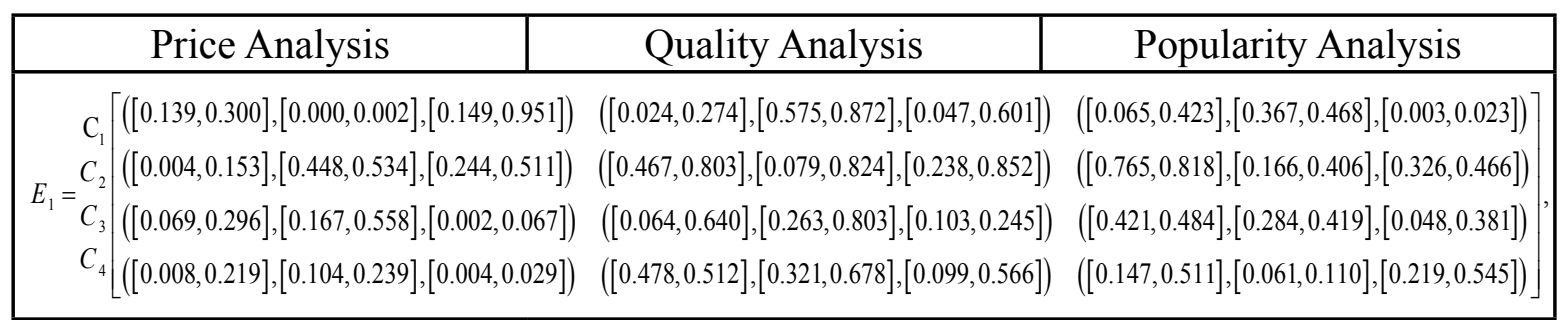


Step (3). By using eq. 4 the function matrix $S$ will be calculated as follows:

\begin{tabular}{|c|c|c|c|}
\hline & $D_{1}$ & $D_{2}$ & $D_{3}$ \\
\hline$C_{1}$ & 0.117 & 0.299 & $0.259]$ \\
\hline$C_{2}$ & 0.374 & 0.212 & 0.175 \\
\hline$C_{3}$ & 0.350 & 0.461 & 0.203 \\
\hline$C_{4}$ & 0.521 & 0.294 & 0.124 \\
\hline
\end{tabular}

Step (4). Based on the ranking method presented in Definition 8, the preference vectors $L^{1}, L^{2}$ and $L^{3}$ of DMs $D_{1}, D_{2}$ and $D_{3}$ can get as follows:

\begin{tabular}{|c|c|c|c|}
\hline$C_{1}$ & $C_{2}$ & $C_{3}$ & $C_{4}$ \\
\hline$L^{1}=[4$ & 2 & 3 & $1]$, \\
\hline$C_{1}$ & $C_{2}$ & $C_{3}$ & $\mathrm{C}_{4}$ \\
\hline$L^{2}=[2$ & 4 & 1 & 3 ], \\
\hline$C_{1}$ & $C_{2}$ & $C_{3}$ & $C_{4}$ \\
\hline$L^{3}=[1$ & 3 & 2 & $4]$, \\
\hline
\end{tabular}

Step (5). In this step the aggregated group evaluating value $d_{i}$ of each alternative with respect to three DMs $D_{1}, D_{2}$ and $D_{3}$ can be calculated as follows:

$d_{i}^{(r)}=\sum_{p=1}^{k}\left(W_{p}^{(r)} \times S\left(G_{i p}\right)\right)(i=1,2,3)$ since

$d_{1}^{(1)}=0.225, d_{2}^{(1)}=0.254, d_{3}^{(1)}=0.338$, and

$d_{4}^{(1)}=0.313$.

In this respect the group preference vector $Q$ can be generated as follows:

$Q=\left[\begin{array}{cccc}C_{1} & C_{2} & C_{3} & C_{4} \\ 4 & 3 & 1 & 2\end{array}\right]$.

Step (6). Given that $Q_{1}=L_{1}^{1}=4, Q_{2}=L_{2}^{3}=3$ and $Q_{3}=L_{3}^{2}=1$, then

$S\left(Q, L^{1}\right)=[4-(4-1)]=1$,

$S\left(Q, L^{2}\right)=[4-(1-1)]=4$, and

$S\left(Q, L^{3}\right)=[4-(3-1)]=2, \quad$ respectively can

be yielded.

Step (7). Based on the similarity degrees calculated in the last step, the following is obtained:

$$
\begin{aligned}
& X_{1}=\frac{S\left(Q, L^{1}\right)}{S\left(Q, L^{1}\right)+S\left(Q, L^{2}\right)+S\left(Q, L^{3}\right)}=\frac{1}{7}=0.143, \\
& X_{2}=\frac{S\left(Q, L^{2}\right)}{S\left(Q, L^{1}\right)+S\left(Q, L^{2}\right)+S\left(Q, L^{3}\right)}=\frac{4}{7}=0.571, \\
& X_{3}=\frac{S\left(Q, L^{3}\right)}{S\left(Q, L^{1}\right)+S\left(Q, L^{2}\right)+S\left(Q, L^{3}\right)}=\frac{2}{7}=0.286 .
\end{aligned}
$$

Therefore, based on eq. 15

$$
B^{(1)}=0.143 \times \frac{1}{3}+0.571 \times \frac{1}{3}+0.286 \times \frac{1}{3}=0.333 .
$$

Because $B^{(1)}<0.990$, it will go to the next step.

Step (8). Now we have:

$t_{1}^{2}=\frac{1}{3} \times(1+0.143)=0.381$,

$t_{2}^{2}=\frac{1}{3} \times(1+0.571)=0.523$, and

$t_{3}^{2}=\frac{1}{3} \times(1+0.286)=0.428$.

Therefore, based on eq. 17 the weights of DMs $D_{1}, D_{2}$ and $D_{3}$ in the second round will be calculated as follows:

$$
\begin{aligned}
& W_{1}^{(2)}=\frac{0.381}{0.381+0.523+0.428}=0.286, \\
& W_{2}^{(2)}=\frac{0.523}{0.381+0.523+0.428}=0.393, \\
& W_{3}^{(2)}=\frac{0.428}{0.381+0.523+0.428}=0.714 .
\end{aligned}
$$

let $r=r+1$ and return to step 5 .

Finally, after eight repeats this method will be stopped in $B^{(8)}=0.9948$, where in this round we have $d_{1}^{(8)}=0.299, d_{2}^{(8)}=0.212, d_{3}^{(8)}=0.459$, $d_{4}^{(8)}=0.293$. Because $d_{3}^{(8)}>d_{1}^{(8)}>d_{4}^{(8)}>d_{2}^{(8)}$, so we can see that the preference order of our alternatives is $\left(C_{3}>C_{1}>C_{4}>C_{2}\right)$. Thus, the manufacturing company $C_{3}$ is the best company among other existing companies based on group multi-attribute decision-making.

The effectiveness of the method presented above can be illustrated by comparison with other existing methods based on autocratic multicriteria decision making. In this sense, it can be noticed that in comparison with other proposed methods that only consider crisp or incomplete information the presented method can also handle indeterminate and inconsistent information in realworld situations. In other words, in real life, the decision-making process implies to agree with something, to be unsure and to disagree with something, so this drawback was approached through the above-mentioned method by using neutrosophic sets and values.

\section{Conclusion}

In this paper, in order to deal with indeterminacy in real decision-making problems, a new method 
for group multi-attribute decision-making based on interval neutrosophic sets has been proposed. By applying this method, the evaluating value of each attribute with respect to the corresponding alternative given by decision-makers is represented by an interval neutrosophic value. In other words, interval neutrosophic sets can give us an additional ability to handle problems with incomplete, uncertain imprecise and inconsistent information related to engineering applications and real meaningful science activities.

\section{REFERENCES}

1. Atanassov, K. (1986). Intuitionistic fuzzy sets, Fuzzy Sets and Systems, 20(1), 87-96.

2. Atanassov, K \& Gargov, G. (1989). Intervalvalued intuitionistic fuzzy sets, Fuzzy Sets and Systems, 31(3), 343-349.

3. Ben-Arieh, D. \& Chen, Z. (2006). Linguisticlabels aggregation and consensus measure for autocratic decision making using group recommendations, IEEE Transactions on Systems, Man, and Cybernetics-Part A: Systems and Humans, 36(3), 558-568.

4. Chen, S. M. \& Tsai, B. H. (2015). Autocratic decision making using group recommendations based on the OWA operator and correlation coefficients, Information Sciences, 290, 106-119.

5. Kou, G., Chao, X., Peng, Y., Xu, L. \& Chen, Y. (2017). Intelligent Collaborative Support System for AHP-Group Decision Making, Studies in Informatics and Control, 26(2), 131-142. DOI: 10.24846/v26i2y201701

6. Nayagam, V. L. G., Muralikrishnan, S. \& Sivaraman, G. (2011). Multi-criteria decisionmaking method based on interval-valued intuitionistic fuzzy sets, Expert Systems with Applications, 38(3), 1464-1467.

7. Parreiras, R. O., Ekel, P. Y. \& Martini, J. S. C. \& Palhares, R. M. (2010). A flexible consensus scheme for multicriteria group decision making under linguistic assessments, Information Sciences, 180(7), 1075-1089.

8. Pedrycz, W. \& Song, M. (2011). Analytic hierarchy process (AHP) in group decision making and its optimization with an allocation of information granularity, IEEE Transactions on Fuzzy Systems, 19(3), 527-539.

9. Pérez, I. J., Cabrerizo, F. J. \& HerreraViedma, E. (2010). A mobile decision support system for dynamic group decisionmaking problems, IEEE Transactions on Systems, Man, and Cybernetics-Part A: Systems and Humans, 40(6), 1244-1256.

10. Rădulescu, C. Z. \& Rădulescu, I. C. (2017). An Extended TOPSIS Approach for Ranking Cloud Service Providers, Studies in Informatics and Control, 26(2), 183-192. DOI: $10.24846 / v 26 i 2 y 201706$

11. Smarandache, F. (2004). A geometric interpretation of the neutrosophic set-A generalization of the intuitionistic fuzzy set, arXiv preprint math $/ 0404520$.

12. Smarandache, F. (2005). A generalization of the intuitionistic fuzzy set, International journal of Pure and Applied Mathematics, 24(3), 287-297.

13. Smarandache, F. (2005). A Unifying Field in Logics: Neutrosophic Logic. Neutrosophy, Neutrosophic Set, Neutrosophic Probability: Neutrosophic Logic. Neutrosophy, Neutrosophic Set, Neutrosophic Probability. Rehoboth, Infinite Study.

14. Smarandache, F. (1999). A Unifying Field in Logics: Neutrosophic Logic. Philosophy, 1-141. American Research Press.

15. Smolikova, R. \& Wachowiak, M. P. (2002). Aggregation operators for selection problems, Fuzzy Sets and Systems, 131(1), 23-34.

16. Wang, H., Smarandache, F., Sunderraman, R. \& Zhang, Y.-Q. (2005). An interval neutrosophic sets and logic: theory and applications in computing. Neutrosophic book series, 5, Hexis.

17. Wang, H., Smarandache, F., Zhang, Y. - Q. \& Sunderraman, R. (2010). Single valued neutrosophicsets, Multispace \& Multistructure. Neutrosophic Transdisciplinarity $\quad(100$ Collected Papers of Sciences), 4, 410-413.

18. Wang, Y. M. \& Elhag, T. M. (2007). A fuzzy group decision making approach for bridge risk assessment, Computers \& Industrial Engineering, 53(1), 137-148.

19. Zadeh, L. A. (1965). Fuzzy sets, Information and control, 8(3), 338-353.

20. Zadeh, L. A. (1975). The concept of a linguistic variable and its application to approximate reasoning - I, Information sciences, 8(3), 199-249. 